\title{
Regular 4-polytopes from the Livingstone graph of Janko's first group
}

\author{
Michael I. Hartley • Isabel Hubard • \\ Dimitri Leemans
}

Received: 17 May 2010 / Accepted: 7 June 2011 / Published online: 20 July 2011

(C) Springer Science+Business Media, LLC 2011

\begin{abstract}
The Janko group $J_{1}$ has, up to duality, exactly two regular rank four polytopes, of respective Schläfli types $\{5,3,5\}$ and $\{5,6,5\}$. The aim of this paper is to give geometric constructions of these two polytopes, starting from the Livingstone graph.
\end{abstract}

Keywords Regular abstract polytopes · First group of Janko · Livingstone graph

\section{Introduction}

In $[10,11]$, Janko constructed a new sporadic simple group, now called $J_{1}$, of order 175560 as a subgroup of the linear group GL(7,11). Livingstone gave in [13] a primitive permutation representation of degree 266 of $J_{1}$, this being the smallest number of points on which $J_{1}$ can be represented. The underlying geometric structure that Livingstone used is now called the Livingstone graph. We refer to Sect. 3 for a detailed discussion on this graph. Most papers that have been written on geometries for $J_{1}$ deal with this graph (see the introduction of [6] for more details and references).

\footnotetext{
M.I. Hartley

DownUnder GeoSolutions, 76 Kings Park Road, West Perth 6005, Australia

e-mail: mikeh@dugeo.com

I. Hubard $(\bowtie)$

Instituto de Matemáticas, UNAM, Área de la Investigación científica, Circuito exterior, Ciudad Universitaria, México 04510, Mexico

e-mail: hubard@matem.unam.mx

D. Leemans

Département de Mathématiques, Université Libre de Bruxelles, C.P. 216, Boulevard du Triomphe 1050, Bruxelles

e-mail: dleemans@ulb.ac.be
} 
More recently, Hartley and Leemans have constructed the universal locally projective polytope $\mathcal{U}$ of type $\{5,3,5\}$ with icosahedral vertex-figures in [7]. They found out that its automorphism group is $J_{1} \times L_{2}(19)$. In fact, they both discovered independently the polytope of type $\{5,3,5\}$ for $J_{1}$ which appears as one of the two nontrivial regular quotients of $\mathcal{U}$. They also constructed, using a Petrie-like construction detailed in [9], a polytope of type $\{5,6,5\}$ whose automorphism group is $J_{1}$, as well as four other thin regular geometries which are not polytopal [8].

As mentioned by Peter McMullen in his review of [7] (see review number 1071.51013 in Zentralblatt), Hartley and Leemans did not give any geometric construction of the $\{5,3,5\}$-polytope for $J_{1} \times L_{2}(19)$ or $J_{1}$. The aim of this paper is to fill this gap. We give such geometric constructions for the polytopes of type $\{5,3,5\}$ and $\{5,6,5\}$ on which $J_{1}$ acts as a regular automorphism group using incidence geometry. Once we have the construction for the $\{5,3,5\}$-polytope for $J_{1}$, it is easy to obtain such a construction for $\mathcal{U}$, using a mixing operation. The constructions make intense use of the knowledge of the Livingstone graph. Moreover, once we have the geometric constructions of the $\{5,3,5\}$ - and $\{5,6,5\}$-polytope, using Construction 5.1 of [12], we can construct the four remaining thin geometries of $J_{1}$ given in [8] as already mentioned in the latter paper.

In Sect. 2, we recall the basic definitions and notation needed to understand this paper. In Sect. 3, we recall known facts about the Livingstone graph $\mathcal{L}$ and describe pentagonal families and Petersen graphs appearing as subgraphs of $\mathcal{L}$. We use these objects to construct the $\{5,3,5\}$-polytope in Sect. 4 and the $\{5,6,5\}$-polytope in Sect. 5.

\section{Definitions and notation}

In this section, we briefly review the basic concepts used throughout the paper. The section is divided into three short subsections. In the first one, we give the notation we shall use for graphs. In the second one, we deal with incidence geometries and abstract polytopes. The last subsection shall cover the basics on Janko's first group.

\subsection{Graphs}

Throughout this paper, $\mathcal{G}=(V(\mathcal{G}), E(\mathcal{G}))$ shall denote a graph with vertex set $V(\mathcal{G})$ and edge set $E(\mathcal{G})$. Given a vertex $v \in V(\mathcal{G})$, we denote the neighborhood of $v$ by $N(V)$, that is, the set of all vertices $u \in V(\mathcal{G})$ such that $\{u, v\}$ is an edge of $\mathcal{G}$; we further denote by $\Gamma_{i}^{v}$ the set of all vertices of $\mathcal{G}$ that are at distance $i$ from $v$ (and shall omit the $v$ when it is clear from the context). In particular, $\Gamma_{0}^{v}=\{v\}$ and $\Gamma_{1}^{v}=N(V)$. The distance between two vertices $u, v \in V(\mathcal{G})$ is denoted by $d(u, v)$.

The automorphism group of $\mathcal{G}$, that is, the set of all the bijections of the vertex set that preserve the edge set, is denoted by $\operatorname{Aut}(\mathcal{G})$. Given a subgraph $\mathcal{H}$ of $\mathcal{G}$, $\operatorname{Stab}_{\text {Aut }(\mathcal{G})}\{\mathcal{H}\}$ denotes the set stabilizer of $\mathcal{H}$ in $\operatorname{Aut}(\mathcal{G})$, while $\operatorname{Stab}_{\text {Aut }(\mathcal{G})}[\mathcal{H}]$ denotes the pointwise stabilizer of $\mathcal{H}$ in $\operatorname{Aut}(\mathcal{G})$.

We recall that a (connected) graph $\mathcal{G}$ of diameter $d$ is a distance-regular graph if there exist integers $b_{i}, c_{i}, i=0,1, \ldots, d$, such that, for any two vertices $u, v \in V(\mathcal{G})$ 
with $d(u, v)=i$, there are exactly $c_{i}$ neighbors of $v$ in $\Gamma_{i-1}^{u}$ and $b_{i}$ neighbors of $v$ in $\Gamma_{i+1}^{u}$ (where we set that $\Gamma_{-1}^{w}=\Gamma_{d+1}^{w}:=\emptyset$, for every vertex $w$ ). Note that in particular a distance-regular graph $\mathcal{G}$ is regular with degree $b_{0}$, and that $c_{1}=1$. For each vertex $v \in V(\mathcal{G})$ and $0 \leq i \leq d$, the subgraph $\Gamma_{i}^{v}$ is also a regular graph and has degree $b_{0}-b_{i}-c_{i}$. The numbers $b_{i}, c_{i}(0 \leq i \leq d)$ are called the intersection numbers and the array $\left\{b_{0}, b_{1}, \ldots, b_{d-1} ; c_{1}, c_{2}, \ldots, c_{d}\right\}$, is called the intersection array of $\mathcal{G}$.

Given a vertex and edge transitive graph $\mathcal{G}$, the distance-distribution or collinearity diagram of $\mathcal{G}$ is the graph whose vertices are the double cosets $H x H$, for $x \in \operatorname{Aut}(\mathcal{G})$, where $H$ is the stabilizer of a fixed vertex of $\mathcal{G}$, with directed edge labeled $\alpha$ from $H x H$ to $H y H$ if their are precisely $\alpha$ cosets of the form $h y H$ for $h \in H$ adjacent to $x H$ in $\mathcal{G}$. It is common to denote the cardinality of each double coset inside the vertex of the collinearity diagram. Note that given the collinearity diagram of a graph, one can read the valency of the vertices, the diameter and girth of the graph.

\subsection{Incidence geometries}

Abstract regular polytopes, string $C$-groups and thin regular residually connected incidence geometries with a linear Buekenhout diagram are in one-to-one correspondence. In this paper, we decide to take the incidence geometry point of view since our aim is to construct the two polytopes mentioned in the introduction as incidence geometries. The interested reader can find the basic theory of regular abstract polytopes in [14].

Most of the following ideas arise from [19] (see also [4], Chap. 3 or [15]).

An incidence structure is a 4-tuple $\Gamma=(X, \sim, t, I)$ where $X$ and $I$ are sets of objects, $t: X \rightarrow I$ is a type function and $\sim$ is a symmetric incidence relation on $X$ such that two objects of the same type are incident if and only if they are equal. The elements of $X$ are called the elements of $\Gamma$ and the elements of $I$ are called the types. The rank of $\Gamma$ is the cardinality of $I$. A flag is a set of pairwise incident elements of $\Gamma$ and a chamber is a flag of type $I$. The rank of a flag is its cardinality. An incidence structure $\Gamma$ is an incidence geometry or geometry provided that every flag is contained in a chamber. Moreover, we say that $\Gamma$ is thin provided that every flag of corank 1 is contained in exactly two chambers.

The residue of a flag $F$ of $\Gamma$ is the incidence structure $\left(X_{F}, \sim_{F}, t_{F}\right)$ over the set of types $I \backslash t(F)$ where $X_{F}$ is the set of elements of $\Gamma$ not in $F$ and incident to $F$. Moreover, $\sim_{F}$ and $t_{F}$ are the restrictions of $\sim$ and $t$ to $X_{F}$ and $I \backslash t(F)$. If $\Gamma$ is a geometry, then obviously $\Gamma_{F}$ is also a geometry. A geometry $\Gamma$ is residually connected provided that every residue of rank at least two of $\Gamma$ has a connected incidence graph.

An automorphism of $\Gamma$ is a bijection of $X$ that preserves the types and the incidence. The set of all automorphisms is a group, the automorphism group $\operatorname{Aut}(\Gamma)$. Let $G \leq \operatorname{Aut}(\Gamma)$ be a group of automorphisms of $\Gamma$. We say that $G$ acts flag-transitively on $\Gamma$ (or that $\Gamma$ is flag-transitive) provided that $G$ acts transitively on all chambers of $\Gamma$, hence also on all flags of any given type $J$ where $J$ is a subset of $I$. If we do not make precise what $G$ is, we assume $G=\operatorname{Aut}(\Gamma)$. 
Let $\Gamma(X, \sim, t, I)$ be a flag-transitive incidence geometry and $G=\operatorname{Aut}(\Gamma)$. Take a chamber $C$ of $\Gamma$. For each subset $J$ of $I$, denote by $G_{J}$ the stabilizer of the flag $F \subseteq C$ of type $J$. Obviously, $G_{\emptyset}=G$ and $G_{I}$ is the stabilizer of a chamber, usually called the Borel subgroup of $\Gamma$. The subgroup lattice of $\Gamma$ is the set of subgroups $\left\{G_{J}: J \subseteq I\right\}$ ordered by inclusion.

If $\Gamma$ is a flag-transitive geometry and its Borel subgroup is the identity, we say that $G$ acts regularly on $\Gamma$.

We refer to [4], Chap. 3, for the definition of Buekenhout diagram (or diagram for short) of a geometry. We say that a diagram is linear if two of its vertices are of degree 1 , all the others are of degree 1 or 2 and it is connected.

We define the (coset) pre-geometry $\Gamma=\Gamma\left(G,\left(G_{i}\right)_{i \in I}\right)$ as follows. The set $X$ of elements of $\Gamma$ consists of all cosets $g G_{i}, g \in G, i \in I$. We define an incidence relation $\sim$ on $X$ by:

$$
g_{1} G_{i} \sim g_{2} G_{j} \quad \text { iff } \quad g_{1} G_{i} \cap g_{2} G_{j} \quad \text { is non-empty in } G .
$$

Theorem 2.1 [1] Let $G$ be a group, I a finite set, and $\mathcal{F}=\left(G_{i}\right)_{i \in I}$ a family of subgroups of $G$. Assume that

(i) For each subset $J$ of I of corank at least $2, G_{J}=\left\langle G_{J \cup\{i\}}: i \in I \backslash J\right\rangle$, and

(ii) The connected components of the diagram of $\Gamma=\Gamma\left(G,\left(G_{i}\right)_{i \in I}\right)$ are strings.

Then

(a) $G$ is flag-transitive on $\Gamma$;

(b) $\Gamma$ is residually connected.

A similar theorem, in terms of incidence complexes as opposed to incidence geometries, can be found in [18].

\subsection{Janko's first group}

As pointed out in the introduction, in [10, 11], Janko constructed a new sporadic simple group, now called $J_{1}$, of order 175560 as a subgroup of the linear group GL(7, 11). This group is the only simple group with abelian 2-Sylow subgroups and with an involution whose centralizer is isomorphic to the direct product of the group of order two and the alternating group $A_{5}$ of order 60 . The entire subgroup pattern of $J_{1}$ was first given by Francis Buekenhout in [3]. In 1985, in a private communication to Buekenhout, Pahlings corrected that pattern. It is now implemented in the computer package GAP[17] as a table of marks and directly available in MAGMA using the "SubgroupLattice" function. In our discussion of the Livingstone graph, we shall make use the subgroup lattice of $J_{1}$. A picture of it is available in [6]. We also provide a picture of this subgroup lattice in Fig. 11.

\section{On the Livingstone graph}

The geometry of the first group of Janko, $J_{1}$, was first described by Livingstone [13] as a permutation representation of $J_{1}$ on 266 vertices. Such a description is often known as the Livingstone graph and the properties of it can be found in $[2,13,16]$, for 
Fig. 1 The collinearity diagram of the Livingstone graph

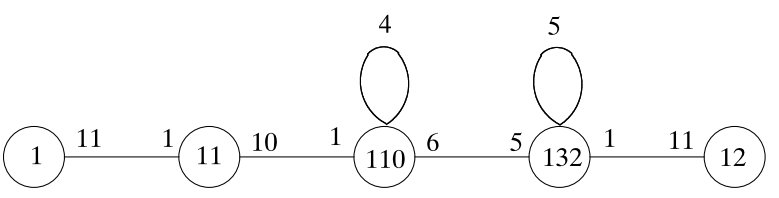

example. In this section, we review some aspect of this graph that will be of great use for us in the next sections, and refer the reader to the above references for some details. We remark that most of the following discussion, in particular Propositions 3.1, 3.2 and 3.3, can be found in [2]; however, we insist on giving some details here as they will be of great help in our understanding when constructing the polytopes.

Let $\mathcal{G}$ be the Livingston graph on 266 vertices and let $G=\operatorname{Aut}(\mathcal{G}) \cong J_{1}$. The graph $\mathcal{G}$ is an 11-regular, 2-arc-transitive graph such that for each $v \in V(\mathcal{G})$, $\operatorname{Stab}_{G}(v) \cong L_{2}(11)$. Moreover, $\operatorname{such} \operatorname{Stab}_{G}(v)$ acts transitively on the 11 vertices of the neighborhood $N(v)$ of $v$. The intersection array of the Livingstone graph is $\{11,10,6,1 ; 1,1,5,11\}$, implying that its collinearity diagram is as in Fig. 1. In particular, the stabilizer of a vertex $v$ has five orbits on $V(\mathcal{G})$, of respective lengths 1 , $11,110,132$ and 12; the elements of the orbit of length 11 are all at distance 1 from $v$, the ones in the orbit of length 110 are at distance 2, and so on. Let us call $\Gamma_{i}^{v}$ the set of vertices at distance $i$ from $v$ (and we shall omit the $v$ when it is clear from the context). From the collinearity diagram, we further derive that the girth of $\mathcal{G}$ is five.

\subsection{The Petersen graphs of $\mathcal{G}$}

As we shall see in this section, there are subgraphs in $\mathcal{G}$ isomorphic to the Petersen graph.

\subsubsection{The pentagons}

Let $v_{0} \in V(\mathcal{G})$, and consider $\Gamma_{i}:=\Gamma_{i}^{v_{0}}$. From Fig. 1, one can see that there are $\frac{11 \times 10 \times 4}{2}=220$ pentagons with $v_{0}$ as a distinguished vertex. Furthermore, these pentagons have exactly two vertices in $\Gamma_{1}$ and the remaining two vertices in $\Gamma_{2}$. Note that the vertex-transitivity of $\mathcal{G}$ tells us now that, in fact, we can find exactly $\frac{266 \times 220}{5}=2926 \times 4$ pentagons in $\mathcal{G}$. In fact, by [2], we have that these pentagons belong to two different orbits under $G$, one with 2926 of them, and the other one with $8778=2926 \times 3$ pentagons. We shall call these two pentagon orbits white (and denote it by $\mathcal{W}$ ) and orange (and denote it by $\mathcal{O}$ ), respectively.

Let $v_{0}, v_{1}, v_{2}$ be a 2 -arc of $\mathcal{G}$ with $v_{0}$ adjacent to $v_{1}$ and $v_{1}$ adjacent to $v_{2}$. For every subgrah $H$ of $G$, let $\operatorname{Stab}_{G} H$ denote the stabilizer of $H$ as a subgraph and let $\operatorname{Stab}_{G}[H]$ denote the pointwise stabilizer of $H$. Then $\operatorname{Stab}_{G}\left\{v_{0}, v_{1}\right\} \cong 2 \times A_{5}$, $\operatorname{Stab}_{G}\left[v_{0}, v_{1}\right] \cong A_{5}$ and $\operatorname{Stab}_{G}\left[v_{0}, v_{1}, v_{2}\right] \cong S_{3}$ (see [16]). Consider all the pentagons of $\mathcal{G}$ that have vertices $v_{0}, v_{1}$ and $v_{2}$ : as there are four vertices adjacent to $v_{2}$ at distance two from $v_{0}$, there are exactly four such pentagons; call the set of these four pentagons $\Omega$. We shall now see in which orbit these pentagons are. First note that given $v_{0}, v_{1}, v_{2}$, for each $v \in N\left(v_{2}\right) \cap \Gamma_{2}$, there is a unique $u \in \Gamma_{1}$ such that $v_{0}, v_{1}, v_{2}, v, u$ form a pentagon. Hence, the way $S_{3}\left(\cong \operatorname{Stab}_{G}\left[v_{0}, v_{1}, v_{2}\right]\right)$ acts on $\Omega$ is equivalent to the way $S_{3}$ acts on $N\left(v_{2}\right) \cap \Gamma_{2}$. 
Fig. 2 Pentagons in $\Omega$

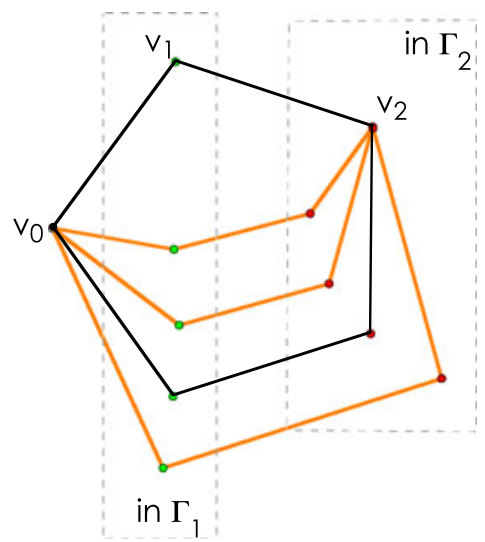

Since the action of $G$ on $\mathcal{G}$ is 2-arc-transitive, $\operatorname{Stab}_{G}\left[v_{0}, v_{1}\right]\left(\cong A_{5}\right)$ acts transitively on the 10 vertices of $\Gamma_{1} \backslash\left\{v_{1}\right\}$. It is well-known that there is only one way in which $A_{5}$ acts transitively on 10 points, namely the way $A_{5}$ acts on the vertices of a Petersen graph. Furthermore, there is only one conjugacy class of subgroups isomorphic to $S_{3}$ in $A_{5}$ and $A_{5}$ acts on the 10 points as the stabilizer of one of the vertices of a Petersen graph: it has three orbits on them, one of length one (the fixed vertex), one of length three (its three adjacent vertices) and the last one of length six (the remaining six vertices of the Petersen graph). This implies that $\operatorname{Stab}_{G}\left[v_{0}, v_{1}, v_{2}\right]$ has exactly three orbits on $\Gamma_{1} \backslash\left\{v_{1}\right\}$, of lengths one, three and six. As each $v \in N\left(v_{2}\right) \cap \Gamma_{2}$ is in correspondence with a vertex $u \in \Gamma_{1} \backslash\left\{v_{1}\right\}$, the action of $\operatorname{Stab}_{G}\left[v_{0}, v_{1}, v_{2}\right] \cong S_{3}$ on $\Gamma_{1} \backslash\left\{v_{1}\right\}$ determines the action of $S_{3}$ on $N\left(v_{2}\right) \cap \Gamma_{2}$, that is, $S_{3}$ should have two orbits on $N\left(v_{2}\right) \cap \Gamma_{2}$ (and hence on $\Omega$ ) of lengths one and three, respectively. A counting argument now gives us that out of the four pentagons of $\Omega$ one is white, while the other three are orange, see Fig. 2.

Consider an orange pentagon. Since its orbit under $G$ has 8778 elements, its stabilizer must be of order 20. Taking a look at the subgroup lattice of $J_{1}$, we see that there is only one conjugacy class of groups of order 20 in $J_{1}$, and such groups are isomorphic to $2 \times D_{10} \cong D_{20}$. That is, the stabilizer of a pentagon in $\mathcal{O}$ is isomorphic to $2 \times D_{10}$. Let us denote by $\mathcal{C}_{\mathcal{O}}$ the conjugacy class of subgroups $2 \times D_{10}$ that stabilize a pentagon of $\mathcal{O}$.

Let now $P:=\left\{v_{0}, v_{1}, v_{2}, v_{3}, v_{4}\right\} \in \mathcal{W} \cap \Omega$. Since $|\mathcal{W}|=2926,\left|\operatorname{Stab}_{G} P\right|=60$, and there are three different conjugacy classes of subgroups of $J_{1}$ with 60 elements. Two such conjugacy classes contain subgroups isomorphic to $A_{5}$, while the other one contains subgroups isomorphic to $S_{3} \times D_{10}$. Note that since $\mathcal{G}$ is 2-arc-transitive, there exist two automorphisms $\rho$ and $\sigma$ of $\mathcal{G}$ sending the 2-arc $v_{0}, v_{1}, v_{2}$ to $v_{0}, v_{4}, v_{3}$ and $v_{1}, v_{2}, v_{3}$, respectively. Thus $\langle\rho, \sigma\rangle \cong D_{10}$ is a subgroup of $\operatorname{Stab}_{G} P$. On the other hand, we knew that the orbit of length one of $\operatorname{Stab}_{G}\left[v_{0}, v_{1}, v_{2}\right]$ in $\Omega$ is precisely $P$. Therefore, $\operatorname{Stab}_{G}\left[v_{0}, v_{1}, v_{2}\right] \leq \operatorname{Stab}_{G}[P] \leq \operatorname{Stab}_{G} P$ and $\operatorname{Stab}_{G} P \cong S_{3} \times D_{10}$. Let us denote by $\mathcal{C}_{\mathcal{W}}$ the conjugacy class of subgroups $S_{3} \times D_{10}$ that stabilize a pentagon of $\mathcal{W}$.

Furthermore, each stabilizer of an orange pentagon is a subgroup of a subgroup $S_{3} \times D_{10}$, the latter being the stabilizer of a white pentagon. Moreover, each element 
Fig. 3 The pentagon $P$, together with $\Gamma_{2} \cap N\left(v_{2}\right)$ and $\Gamma_{2} \cap N\left(v_{3}\right)$

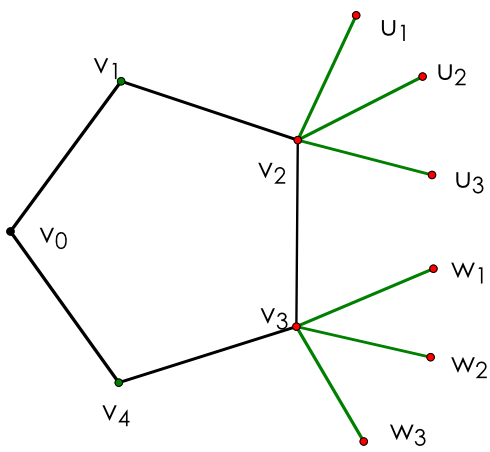

of $\mathcal{C}_{\mathcal{W}}$ contains exactly three elements of $\mathcal{C}_{\mathcal{O}}$. In other words, the stabilizer of every pentagon in $\mathcal{O}$ stabilizes exactly one pentagon in $\mathcal{W}$, while the stabilizer of each pentagon in $\mathcal{W}$ contains the stabilizers of three pentagons in $\mathcal{O}$.

\subsubsection{The Petersen graphs}

Consider now $\Gamma_{2} \cap N\left(v_{2}\right)=:\left\{u_{1}, u_{2}, u_{3}, v_{3}\right\}$ and $\Gamma_{2} \cap N\left(v_{3}\right)=:\left\{w_{1}, w_{2}, w_{3}, v_{2}\right\}$ (see Fig. 3). Recall that, for $P=\left\{v_{0}, \ldots, v_{4}\right\}$, there exists $\rho \in \operatorname{Stab}_{G}(P)$ sending $v_{0}, v_{1}, v_{2}$ to $v_{0}, v_{4}, v_{3}$. Such $\rho$ sends $\left\{u_{1}, u_{2}, u_{3}\right\}$ to $\left\{w_{1}, w_{2}, w_{3}\right\}$. On the other hand, each of the $u_{i}$ 's, together with $v_{0}, v_{1}$ and $v_{2}$, determines a pentagon in $\mathcal{O}$, and hence $S_{3} \cong \operatorname{Stab}_{G}\left[v_{0}, v_{1}, v_{2}\right]$ acts transitively on $\left\{u_{1}, u_{2}, u_{3}\right\}$. Since $S_{3}$ and $D_{10}$ commute in $\operatorname{Stab}_{G} P, S_{3}$ also acts transitively on $\left\{w_{1}, w_{2}, w_{3}\right\}$. Moreover, $S_{3}$ acts transitively on the sets $\sigma^{i}\left\{u_{1}, u_{2}, u_{3}\right\}$, for $i=1,2,3,4$ (where we recall that $\sigma \in D_{10} \leq \operatorname{Stab}_{G} P$ is the 'rotation' that sends $v_{0}, v_{1}, v_{2}$ to $\left.v_{1}, v_{2}, v_{3}\right)$. Therefore, $\sigma$ also maps $\left\{u_{1}, u_{2}, u_{3}\right\}$ to $\left\{w_{1}, w_{2}, w_{3}\right\}$.

Now, the set $\left\{u_{1}, u_{2}, u_{3}\right\}^{\langle\sigma\rangle}$ contains 15 vertices, all at distance one from $P$, and with the property that $\operatorname{Stab}_{G} P$ stabilizes them (as a set). Let $H$ be the subgraph of $\mathcal{G}$ on these 15 vertices. Let $x_{i}:=u_{i} \sigma^{3}$, so that $x_{i} \in \Gamma_{1}$ for each $i=1,2,3$, and $S_{3} \cong$ $\operatorname{Stab}_{G}\left[v_{0}, v_{1}, v_{2}\right]$ acts transitively on $\left\{x_{1}, x_{2}, x_{3}\right\}$. We know that for each $i=1,2,3$, $v_{0}, v_{1}, v_{2}$ and $u_{i}$ determine a pentagon in $\mathcal{O}$; call $P_{i}$ such pentagon, and let $a_{i}$ be the remaining vertex of them. (Hence $a_{i} \in \Gamma_{1}$.) Note that since $S_{3} \cong \operatorname{Stab}_{G}\left[v_{0}, v_{1}, v_{2}\right]$ ) acts transitively on $\left\{P_{1}, P_{2}, P_{3}\right\}$, it acts transitively on $\left\{a_{1}, a_{2}, a_{3}\right\}$. But we also know that the orbits of $S_{3}$ on $\Gamma_{1}$ have lengths $1,1,3$, and 6 , implying that $\left\{x_{1}, x_{2}, x_{3}\right\}=$ $\left\{a_{1}, a_{2}, a_{3}\right\}$ (see Fig. 4).

By rotating $\left\{u_{1}, u_{2}, u_{3}\right\}$ and $\left\{x_{1}, x_{2}, x_{3}\right\}$ with $\sigma$, we see that $H$ consists of cycles: either one 15-cycle, or a 5-cycle and a 10-cycle or three 5-cycles. Since $S_{3}$ acts transitively on $\left\{u_{1}, u_{2}, u_{3}\right\}$ and its images under the elements of $\langle\sigma\rangle$, the cycles of $H$ should all have the same length, so that $H$ cannot be the union of a 5-cycle and a 10 -cycle. On the other hand, there exists $\alpha \in S_{3}$ such that $u_{1} \alpha=u_{2}$ and $u_{3} \alpha=u_{3}$, and hence $x_{1} \alpha=x_{2}$ and $x_{3} \alpha=x_{3}$, but no symmetry of a 15-cycle different from the identity fixes two point, implying that $H$ is, in fact, the union of three disjoint pentagons. Without loss of generality, we can say that each of these three pentagons, $P_{1}, P_{2}$ and $P_{3}$, has vertices $\left\{u_{i}, x_{i}, w_{i}, z_{i}, y_{i}\right\}, i=1,2,3$, respectively. 
Fig. 4 The pentagon $P$, $\Gamma_{2} \cap N\left(v_{2}\right)$ and the pentagons $\mathcal{O}$ induced by them

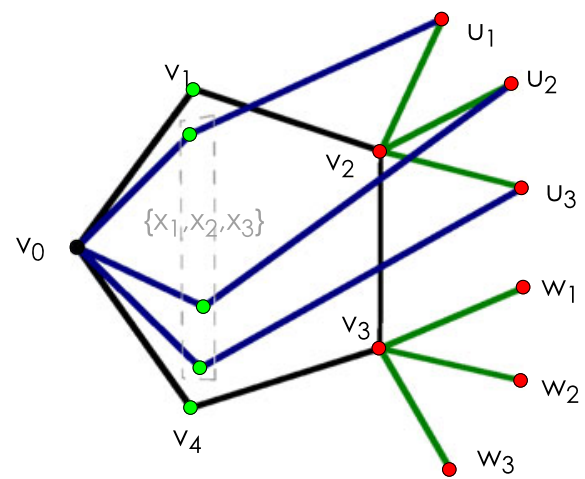

In fact, one may show that the vertices of $P$, together with the vertices of each of the $P_{i}$ give a subgraph $\mathbb{P}_{i}$ of $\mathcal{G}$ on 10 vertices isomorphic to the Petersen Graph, and that $D_{10}<\operatorname{Stab}_{G} P$ stabilizes each of them. We know that the pentagon $P$ in $\mathbb{P}_{i}$ belongs to $\mathcal{W}$. In what follows, we shall find out in which orbit each of the other 11 pentagons of $\mathbb{P}_{i}$ is.

We summarize the above discussion in the following proposition.

Proposition 3.1 [2] Each white pentagon $P$ belongs to three Petersen subgraphs of $\mathcal{G}$; in each of them, the subgraph spanned by the vertices not in $P$ is an orange pentagon.

\subsubsection{The pentagons of the Petersen graphs}

Let us start by considering the three pentagons $P_{1}, P_{2}$ and $P_{3}$. Recall that they belong to the same orbit. Assume that they are in $\mathcal{W}$. Then $\operatorname{Stab}_{G} P_{i} \cong S_{3} \times D_{10}$; furthermore, the $D_{10}$ in $\operatorname{Stab}_{G} P$ actually stabilizes each of the $P_{i}$ 's, while the $S_{3}$ in $S_{3} \times D_{10}$ permutes them. Thus $\operatorname{Stab}_{G} P \cap \bigcap_{1}^{3} \operatorname{Stab}_{G} P_{i} \cong D_{10}$. That is, there is a group isomorphic to $D_{10}$ that is contained in four different $S_{3} \times D_{10}$. However, this yields us to a contradiction in the subgroup lattice of $J_{1}$. The lattice tells us that there are two different classes of subgroups isomorphic to $D_{10}$, both contained in the class of $S_{3} \times D_{10}$ (see Fig. 11).

However, regardless of the class of $D_{10}$ that we take, each such group is contained in exactly one group in the class $S_{3} \times D_{10}$. (Note, however, that each subgroup in the class $S_{3} \times D_{10}$ contains exactly one $D_{10}$ of one of the classes, but three of the other class.) In other words, the $P_{i}$ 's cannot belong to $\mathcal{W}$, and therefore they belong to $\mathcal{O}$. Hence these pentagons correspond precisely to the three $D_{10}$ 's belonging to the same conjugacy class inside $S_{3} \times D_{10} \cong \operatorname{Stab}_{G} P$.

Recall that the pentagons with vertices $v_{0}, v_{1}, v_{2}, u_{i}$ and $x_{i}$ are orange, for each $i=1,2,3$. Moreover, the images of these pentagons under $\langle\sigma\rangle$ are also orange; in particular, the pentagon $Q_{1}$ with vertices $v_{2}, v_{3}, v_{4}, y_{1}$ and $u_{1}$ is in $\mathcal{O}$ (see Fig. 5). Suppose now that the pentagon $Q_{2}$ with vertices $w_{1}, v_{3}, v_{4}, y_{1}$ and $z_{1}$ is in $\mathcal{O}$. Hence there exists an automorphism $\alpha \in G$ that sends $Q_{2}$ to $Q_{1}$. Furthermore, since $\operatorname{Stab}_{G} Q_{1} \cong \operatorname{Stab}_{G} Q_{2} \cong 2 \times D_{10}$, we may assume that $\alpha$ fixes $v_{3}, v_{4}$ and $y_{1}$, while it sends $w_{1}$ and $z_{1}$ to $v_{2}$ and $u_{1}$, respectively. 
Fig. 5 The Petersen graph $\mathbb{P}_{1}$

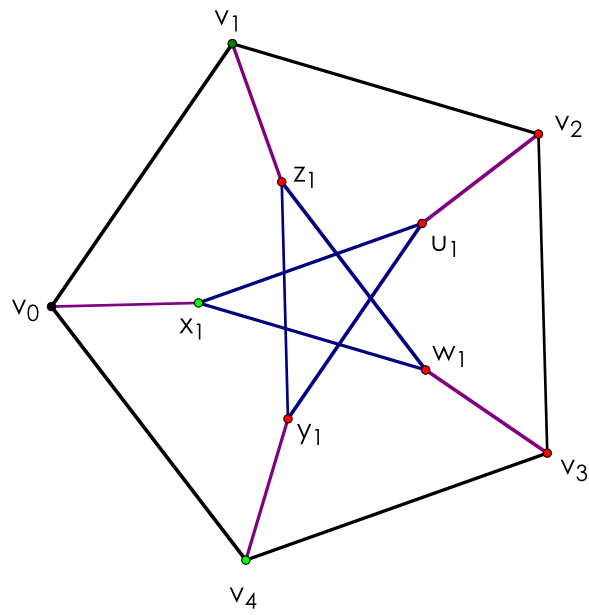

Let us first assume that $\alpha$ is not an involution. Since $\alpha$ fixes $v_{3}, v_{4}$ and $y_{1}$, $\alpha \in \operatorname{Stab}_{G}\left[v_{3}, v_{4}, y_{1}\right] \cong S_{3}$ implying that $\alpha^{3}=i d$. Then $Q_{3}:=Q_{2} \alpha \in \mathcal{O}$. Since $Q_{1}, Q_{2}, Q_{3} \in \mathcal{O}$ share the vertices $y_{1}, v_{3}$ and $v_{4}$, there is an $S_{3}\left(\cong \operatorname{Stab}_{G}\left[y_{1}, v_{3}, v_{4}\right]\right)$ acting transitively on them. We can then choose $\beta \in \operatorname{Stab}_{G}\left[y_{1}, v_{3}, v_{4}\right]$ of order two that sends $Q_{2}$ to $Q_{1}$ and fixes $Q_{3}$. In other words, without loss of generality, we may assume that $\alpha^{2}=i d$.

From this we induce that $v_{1} \alpha$ is a vertex adjacent to both $u_{1}\left(=z_{1} \alpha\right)$ and $w_{1}\left(=v_{2} \alpha\right)$, and since there are no 4-cycles in $\mathcal{G}$, this implies that $v_{1} \alpha=x_{1}$, and so $x_{1} \alpha=v_{1}$. In a similar way, $v_{0} \alpha=v_{0}$. But this is telling us that $\alpha \in G$ sends $P \in \mathcal{W}$ to $\left\{v_{0}, x_{1}, w_{1}, v_{3}, v_{4}\right\} \in \mathcal{O}$, which is a contradiction. Therefore, for each $i=1,2,3$, the pentagon with vertices $v_{3}, v_{4}, y_{i}, z_{i}$ and $w_{i}$ is in $\mathcal{W}$ (together with their images under $\langle\sigma\rangle)$.

In particular, out of the 12 pentagons there are in each Petersen graph, 6 of them are white, while the other 6 are orange. Note then, that given a vertex in a Petersen graph of $\mathcal{G}$, out of the six pentagons that contain it, three of them are in $\mathcal{W}$, while the other three are in $\mathcal{O}$. Furthermore, if $\{u, v\}$ is an edge of a Petersen graph $\mathbb{P}$, then out of the three pentagons in $\mathcal{W} \cap \mathbb{P}$ (resp., $\mathcal{O} \cap \mathbb{P}$ ) that contain $v$, exactly two of them also contain $u$ and hence the edge $\{v, u\}$. This means that there is exactly one pentagon in $\mathcal{W} \cap \mathbb{P}$ and one pentagon in $\mathcal{O} \cap \mathbb{P}$ containing $v$, but not $u$ (or $u$ but not $v$ ).

We summarize the above discussion in the following proposition.

Proposition 3.2 [2] Each Petersen subgraph $\mathbb{P}$ of $\mathcal{G}$ contains 6 white pentagons and 6 orange pentagons. Moreover, given any white pentagon $P$ of $\mathbb{P}$, the vertices of $\mathbb{P}$ not in $P$ span an orange pentagon.

\subsubsection{The stabilizers of the Petersen graphs}

Consider the two orange pentagons $P_{1}:=\left\{x_{1}, w_{1}, z_{1}, y_{1}, u_{1}\right\}$ and $P_{\mathcal{O}}:=\left\{x_{1}, w_{1}, v_{3}\right.$, $\left.v_{4}, v_{0}\right\}$ (see Fig. 5), and let $\gamma \in G$ be such that $P_{1} \gamma=P_{\mathcal{O}}$. Note that $P=\left\{v_{0}, \ldots, v_{4}\right\}$ and $P_{\mathcal{W}}:=\left\{u_{1}, v_{2}, v_{1}, z_{1}, y_{1}\right\}$ are the two white pentagons that are the complement 
of $P_{1}$ and $P_{\mathcal{O}}$, respectively, in the Petersen graph $\mathbb{P}_{1}$. We know that the $\operatorname{Stab}_{G} P_{1} \cong$ $2 \times D_{10}$ stabilizes $\mathbb{P}_{1}$, and since $\gamma \in \operatorname{Stab} \mathbb{P}_{1}$ does not stabilize $P_{1}$, we obtain that $2 \times$ $D_{10}<\operatorname{Stab}_{G} \mathbb{P}_{1}<J_{1}$. The only such class of subgroups of $J_{1}$ is the one containing subgroups isomorphic to $2 \times A_{5}$. Therefore, $\operatorname{Stab}_{G} \mathbb{P}_{1} \cong 2 \times A_{5}$.

Since the automorphism group of a Petersen graph is isomorphic to $S_{5}$, the subgroup $C_{2}:=Z\left(\operatorname{Stab}_{G} \mathbb{P}_{1}\right)$ of $\operatorname{Stab}_{G} \mathbb{P}_{1}$ fixes every point of $\mathbb{P}_{1}$, while the nontrivial elements in $A_{5}$ do not do this, implying that the pointwise stabilizer of $\mathbb{P}_{1}$ is generated by an involution.

On the other hand, it is also known that the stabilizer of an edge of $\mathcal{G}$ is isomorphic to $2 \times A_{5}$. Furthermore, there is only one conjugacy class of subgroups isomorphic to $2 \times A_{5}$ in $J_{1}$, and the orbits of one such subgroup on the 266 vertices of $\mathcal{G}$ have lengths $2,10,20,24,30,60$ and 120 . The orbit of length 10 of the stabilizer of an edge is precisely giving a subgraph of $\mathcal{G}$ isomorphic to the Petersen graph. In fact, given an edge $e=\{u, v\}$, there are 10 white pentagons containing it; the set of vertices in those pentagons that are at distance two from $e$ form a Petersen graph $\mathbb{P}$ with $\operatorname{Stab}_{G}\{u, v\}=\operatorname{Stab}_{G} \mathbb{P}$.

Finally, note that given $e \in E(\mathcal{G})$, and its corresponding Petersen graph $\mathbb{P}$, when we choose a pentagon $P \in \mathcal{W} \cap \mathbb{P}$ and its corresponding complementary $P_{1} \in \mathcal{O} \cap \mathbb{P}$, we have $\operatorname{Stab}_{G} P_{1}=2 \times D_{10}$ acting on the other two Petersen graphs defined by $P$. Therefore, $2 \times D_{10}$ also has an orbit of length four on the vertices of $\mathcal{G}$, which contains the two pairs of points determined by these two other Petersen graphs. Let $\mathbb{P}(\mathcal{G})$ denote the set containing the four-tuples of the type $\left\{P, P_{1}, e,\left\{e_{0}, e_{1}\right\}\right\}$, where $P \in \mathcal{W}$ and $P_{1} \in \mathcal{O}$ determine a Petersen graph, $e$ is the edge related to this Petersen graph, and $\left\{e_{0}, e_{1}\right\}$ are the two edges determined by the other two Petersen graphs on $P$.

We summarize the above discussion in the following proposition.

Proposition 3.3 [2] Given an edge e of $\mathcal{G}$, there exists a Petersen subgraph $\mathbb{P}$, whose vertices are all the vertices at distance two of each vertex of e. Moreover, $\operatorname{Stab}_{G}(e)=$ $\operatorname{Stab}_{G}(\mathbb{P}) \cong 2 \times A_{5}$.

\subsection{A rank two geometry in $\mathcal{G}$}

\subsubsection{The other conjugacy class of subgroups isomorphic to $A_{5}$}

As we saw before, given an edge $e=\{v, u\}$ of $\mathcal{G}$, the pointwise stabilizer of $e$ is isomorphic to $A_{5}$. But by taking a look at the subgroup lattice of $J_{1}$, we see that there are two different conjugacy classes of $A_{5}$ that are subgroups of $L_{2}(11)$. While the conjugacy class corresponding to the pointwise stabilizer of $e$ acts transitively on $N(v) \backslash\{u\}$ (that is, it has two orbits on $N(v)$, one of length one and the other one of length 10), the other class that fixes the vertex $v$, has also two orbits of $N(v)$ but now of lengths five and six, respectively.

Let $v_{1} \in V(\mathcal{G})$ be fixed, and consider an $A_{5}:=H$ acting on its neighborhood, having orbits of lengths five and six; call these two orbits $V_{5}$ and $V_{6}$, respectively. Note that fixing an edge $e$ in which $v_{1}$ is contained gives us an $A_{5}$ not conjugated to $H$. It is well-known (see the subgroup lattice) that the intersection of these two $A_{5}$ 's will depend on whether the vertex $u \in e, u \neq v$ is in $V_{5}$ or on $V_{6}$; in fact, if $u \in V_{5}$, then $\operatorname{Stab}_{G}[e] \cap H \cong A_{4}$, while if $u \in V_{6}$, then $\operatorname{Stab}_{G}[e] \cap H \cong D_{10}$. 


\subsubsection{A helpful rank 2 geometry}

In [5], Buekenhout et al. determine all residually connected, primitive flag-transitive geometries for $L_{2}(11)$. Of our interest is the geometry $\Gamma$ of rank 2, listed as number 7 in Sect. 6.1 of the latter reference. We now describe this rank 2 geometry. Note that this rank 2 geometry can be seen as the unique biplane with 11 points and 11 lines, whose automorphism group is precisely $L_{2}(11)$; the details of the description of such biplane can be found in [15]. This coset geometry, with points and blocks, is such that, given a point $p$ and a block $b$ incident to $p$, the stabilizer of $b$ and the stabilizer of $p$ in $L_{2}(11)$ are both isomorphic to $A_{5}$ but not conjugate in $L_{2}(11)$. The stabilizer of $b$ and $l$ in $L(2,11)$ is isomorphic to $D_{10}$. So $\Gamma$ consists of 11 points, distributed on blocks of six points, in such a way that any two points determine exactly three blocks. As there are 11 points and every block has six points, there are 11 blocks and every point is in six blocks. Moreover, every two blocks intersect in exactly three points. This geometry is unique, up to isomorphism, in the sense that any coset geometry of rank two for $L_{2}(11)$ with $G_{0} \cong G_{1} \cong A_{5}, G_{0} \cap G_{1} \cong D_{10}$ and $G_{0}$ not conjugate to $G_{1}$ in $L_{2}(11)$ is isomorphic to $\Gamma$.

Hence, given a vertex $v_{1}$ of $\mathcal{G}$, the geometry $\Gamma$ precisely describes the 11 subgroups isomorphic to $A_{5}$ in $L_{2}(11)$ that have orbits of length 5 and 6 on $N\left(v_{1}\right)$. That is, the 11 vertices of $N\left(v_{1}\right)$ correspond to the 11 points of $\Gamma$, while the 11 blocks of $\Gamma$ correspond to the 11 subgroups acting on these 11 points. In particular this implies that choosing any two vertices in $N\left(v_{1}\right)$, there are precisely three sets of six elements of $N\left(v_{1}\right)$ in which there is an $A_{5}$ acting transitively.

Let us denote by $\mathbb{V}(\mathcal{G})$ the set consisting of all triples $\left\{v_{1}, V_{5}, V_{6}\right\}$, where $v_{1} \in$ $V(\mathcal{G}), V_{5} \cup V_{6}=N\left(v_{1}\right)$, with $\left|V_{i}\right|=i$ and such that there is an $A_{5}<\operatorname{Stab}_{G} v$ acting transitively on both $V_{5}$ and $V_{6}$.

\subsubsection{Blocks and Petersen graphs}

Let $v_{1} \in V(\mathcal{G})$, and $v_{0}, v_{2} \in N\left(v_{1}\right)$. Let $A, B$ and $C$ be the three blocks of $\Gamma$ determined by $v_{0}$ and $v_{2}$ (so that $N\left(v_{1}\right) \backslash\left\{v_{0}, v_{2}\right\}=A \cup B \cup C$ ). Since the intersection of any two blocks contains exactly three points, we can label the points in $A, B$ and $C$ as follows:

$$
A:=\{b, c, p, q\}, \quad B:=\{a, c, x, y\}, \quad C:=\{a, b, z, w\} .
$$

Note that the 2-arc $\left\{v_{0}, v_{1}, v_{2}\right\}$ determines a unique white pentagon, and this, in turn, gives us exactly three Petersen graphs, say $\mathbb{P}_{0}, \mathbb{P}_{1}, \mathbb{P}_{2}$. As $\left|\mathbb{P}_{i} \cap N\left(v_{1}\right)\right|=3$ and $v_{0}, v_{2} \in \mathbb{P}_{i} \cap N\left(v_{1}\right)$, each of the Petersen graphs intersects $N\left(v_{1}\right) \backslash\left\{v_{0}, v_{2}\right\}$ in exactly one point, giving in this way three special points that belong to $A \cup B \cup C$, one per Petersen graph. In what follows, we shall show that these three points are precisely $a, b$ and $c$.

Now, $\operatorname{Stab}_{G}\left\{v_{0}, v_{1}, v_{2}\right\} \cong 2 \times S_{3}$, where the 2 acts as a reflection of the 2 -arc, while the $S_{3}$ acts as the permutation group on the blocks $A, B$ and $C$. On the other hand, this $S_{3}$ has two orbits on $N\left(v_{1}\right) \backslash\left\{v_{0}, v_{2}\right\}: \Delta_{3}$, of length three and $\Delta_{6}$, of length six (and so it acts transitively on each of them). In particular, this means that no element of $S_{3}$ different than the identity fixes a point of $\Delta_{6}$, while the only element of $S_{3}$ 
that fixes all points of $\Delta_{3}$ is the identity. Moreover, we know that the three points of $N\left(v_{1}\right) \backslash\left\{v_{0}, v_{2}\right\}$ that belong to the Petersen graphs described above are the elements of $\Delta_{3}$ (labeled $z_{1}, z_{2}, z_{3}$ is Sect. 3.1.2).

Consider the stabilizer of the block $A$, isomorphic to $A_{5}$. If we fix not only $A$ but also $v_{0}$ and $v_{2}$, we obtain that $\operatorname{Stab}_{G}\left[A, v_{0}, v_{1}, v_{2}\right] \cong 2=:\langle\alpha\rangle$. Since $\alpha$ fixes both $v_{0}$ and $v_{2}$, it fixes the set of blocks $A, B, C$; and as it fixes $A$, it can either fix both $B$ and $C$ or interchange them.

Let us look at the action of $\alpha$ on the elements of $A$. Suppose that $b \alpha=b$. Then $\alpha$ fixes $B$ and therefore it fixes $C, c$ and $a$. This implies that $a, b, c \notin \Delta_{6}$, so that $a, b, c \in \Delta_{3}$. But the only element of $\operatorname{Stab}_{G}\left[v_{0}, v_{1}, v_{2}\right]$ that fixes all elements of $\Delta_{3}$ is the identity, which is a contradiction. Therefore, $\alpha$ does not fix $b$. In a similar way, $\alpha$ cannot fix $c$. By assuming that $\alpha$ sends $b$ to either $p$ or $q$, we see that, as $b \in B, \alpha$ cannot keep $B$ and $C$ as blocks, which cannot happen, and hence $b \alpha=c$. This now implies that $B \alpha=C$ and that $a \alpha=a$. Then, again, as $\alpha$ fixes $a, a \notin \Delta_{6}$, therefore $a \in \Delta_{3}$.

By now considering the stabilizers of the blocks $B$ and $C$, respectively, we obtain that $b, c \in \Delta_{3}$. Furthermore, fixing a block, say $A$, exactly two of the points of $\Delta_{3}$ are in $A$, while the third one is in the complement of such block. Note that given $v_{0}, v_{1}, v_{2}$ and one of the points in $\Delta_{3}$, the Petersen graph is completely determined.

The above discussion leads us to the following proposition.

Proposition 3.4 Given a vertex $v_{1} \in V(\mathcal{G})$, and any three vertices $i, j, k \in N\left(v_{1}\right)$, there is always either one or two blocks of $\Gamma$ containing them. Furthermore, there are exactly two blocks of $\Gamma$ containing $i, j$ and $k$ if and only if there is a Petersen graph of $\mathcal{G}$ containing the four vertices $v_{1}, i, j, k$.

\subsubsection{Blocks from pentagons and pentagons from blocks}

We first consider a Petersen graph $\mathbb{P}$, with a distinguished pentagon $P_{\mathcal{W}}$ in $\mathcal{W}$ (and hence "complementary" pentagon $P_{\mathcal{O}}$ in $\mathcal{O}$ ). This Petersen graph has an edge $e$ associated to it that is a distance two of each of the points of $\mathbb{P}$. Let $v_{1}, v_{0}$ be the end points of $e$. Note that $\operatorname{Stab}_{G}\left\{P_{\mathcal{O}}\right\} \cong 2 \times D_{10}$, while $\operatorname{Stab}_{G}\left\{P_{\mathcal{O}}, v_{1}\right\} \cong D_{10}$.

To each vertex $w$ of $\mathbb{P}$ we can associate exactly one point $u$ in $N\left(v_{1}\right)$ to it, namely, the vertex in $N\left(v_{1}\right)$ that belongs to the (unique) pentagon in $\mathcal{W}$ spanned by $e$ and $w$. This gives us a partition of $N\left(v_{1}\right) \backslash\{u\}$ into two sets $A$ and $B$, of five vertices each, corresponding to the points in $P_{\mathcal{W}}$ and $P_{\mathcal{O}}$, respectively.

Since $\operatorname{Stab}_{G}\left\{v_{1}, P_{\mathcal{O}}\right\} \cong D_{10}$ fixes both $v_{0}$ and $v_{1}$ and acts transitively on both pentagons $P_{\mathcal{W}}$ and $P_{\mathcal{O}}$, it acts transitively on each of the sets $A$ and $B$. But there is only one conjugacy class of subgroups isomorphic to $D_{10}$ inside $\operatorname{Stab}_{G}[e] \cong A_{5}$, and such class is also contained in the other class of $A_{5}$. That is, $\operatorname{Stab}_{G}\left\{v_{1}, P_{\mathcal{O}}\right\} \cong D_{10}$ can be seen as a subgroup of the $H \cong A_{5}$ in $\operatorname{Stab}_{G}\left\{v_{1}\right\}$. Hence, the two sets $A$ and $B$ correspond to the two sets $V_{5}$ and $V_{6} \backslash\left\{v_{0}\right\}$ (though we don't know if $A$ corresponds to $V_{5}$ or to $\left.V_{6} \backslash\left\{v_{0}\right\}\right)$.

On the other hand, we have the following proposition. 
Proposition 3.5 Let $\left\{v_{1}, V_{5}, V_{6}\right\} \in \mathbb{V}(\mathcal{G})$ (as defined in Sect. 3.2.2) and let $v_{0} \in V_{6}$. Then, the edge $\left\{v_{0}, v_{1}\right\}$ induces a unique element of $\mathbb{P}(\mathcal{G})$.

Proof Let $H \cong A_{5}$ in $\operatorname{Stab}_{G}\left\{v_{1}\right\}$ be such that its orbits on $N\left(v_{1}\right)$ are $V_{5}$ and $V_{6}$, and hence $\operatorname{Stab}_{G}\left[v_{1}, v_{0}\right] \cap H \cong D_{10}=: H^{\prime}$. Then $H^{\prime}$ acts transitively on the five points of $V_{5}$, as well as on the five points of $V_{6} \backslash\left\{v_{0}\right\}$. Consider now the Petersen graph $\mathbb{P}$ associated to $\left\{v_{0}, v_{1}\right\}$ (that is, such that $\left.\operatorname{Stab}_{G} \mathbb{P}=\operatorname{Stab}_{G}\left\{v_{0}, v_{1}\right\}\right)$. The vertices of $\mathbb{P}$ are in one to one correspondence to the vertices of $N\left(v_{1}\right) \backslash\left\{v_{0}\right\}$; hence the vertices of $\mathbb{P}$ get divided into two sets, say $P$ and $Q$, each with five elements, corresponding to the sets $V_{5}$ and $V_{6} \backslash\left\{v_{0}\right\}$, respectively. Note then that the two transitive orbits of $H^{\prime}$ on $\mathbb{P}$ are precisely $P$ and $Q$.

Since a maximal independent set (also called coclique) of a Petersen graph has 4 vertices, any subset of 5 vertices contains an edge. Since $D_{10}$ acts transitively on $P$ and $\mathcal{G}$ has girth 5, the subgraph $P$ must be a cycle. That is, $P$ and $Q$ are disjoint pentagons of $\mathbb{P}$ and $\mathbb{P}$, together with $P$ and $Q$, is the unique element of $\mathbb{P}(\mathcal{G})$ induced by $\left\{v_{0}, v_{1}\right\}$.

\section{A polytope of type $\{5,3,5\}$}

The understanding of the Livingstone graph obtained in the previous section provides us now with enough information to define an incidence structure $\mathcal{Q}=\left(\bigcup_{0}^{3} \mathcal{F}_{i}, \sim, t\right.$, $\{0, \ldots, 3\})$, where $\mathcal{F}_{0}$ is the set of pairs $\{e, \mathbb{P}\}$ where $e$ is an edge of $\mathcal{G}$ and $\mathbb{P}$ is its associated Petersen graph (see Sect. 3.1.4), $\mathcal{F}_{1}$ and $\mathcal{F}_{2}$ are two copies of $\mathbb{P}(\mathcal{G})$ (see also Sect. 3.1.4), and $\mathcal{F}_{3}$ is the set $\mathbb{V}(\mathcal{G})$ defined in Sect. 3.2.3. The type function $t: f_{i} \in \mathcal{F}_{i} \mapsto i$ and the incidence relation $\sim$ is defined as follows.

Let $F_{0}=\{e, \mathbb{P}\} \in \mathcal{F}_{0}, F_{1}=\left\{P, P_{1}, e,\left\{e_{0}, e_{1}\right\}\right\} \in \mathcal{F}_{1}, F_{2}=\left\{P^{\prime}, P_{1}^{\prime}, e^{\prime},\left\{e_{0}^{\prime}, e_{1}^{\prime}\right\}\right\} \in$ $\mathcal{F}_{2}$ and $F_{3}=\left\{v_{1}, V_{5}, V_{6}\right\} \in \mathcal{F}_{3}$. Then,

$$
\begin{aligned}
& F_{3} \sim F_{0} \quad \Leftrightarrow \quad v_{1} \in \mathbb{P} \quad \text { and } \quad\left|V_{6} \cap \mathbb{P}\right|=3 ; \\
& F_{3} \sim F_{1} \quad \Leftrightarrow \quad v_{1} \in P, \quad\left|V_{6} \cap P\right|=2 \quad \text { and } \quad\left|V_{5} \cap P_{1}\right|=1 ; \\
& F_{3} \sim F_{2} \quad \Leftrightarrow \quad v_{1} \in e^{\prime} \quad \text { and } \quad\left|V_{6} \cap e^{\prime}\right|=1 ; \\
& F_{2} \sim F_{0} \quad \Leftrightarrow \quad e^{\prime} \in \mathbb{P}, \quad P_{1}^{\prime} \cap \mathbb{P} \text { is an edge } \\
& F_{2} \sim F_{1} \quad \Leftrightarrow \quad e^{\prime} \in P, \quad\left|V\left(P \cap P_{1}^{\prime}\right)\right|=\left|V\left(P_{1} \cap P^{\prime}\right)\right|=1 ; \\
& F_{1} \sim F_{0} \quad \Leftrightarrow \quad P \in \mathbb{P} \quad \text { and } a \in\left\{e_{0}, e_{1}\right\} .
\end{aligned}
$$

Throughout this paper, we shall refer to the elements of $\mathcal{F}_{i}$ as the $i$-faces of $\mathcal{Q}$. Furthermore, the 0-, 1-, and 3-faces are often called vertices, edges and facets, respectively. Note that the stabilizer of a 0 -face is isomorphic to $2 \times A_{5}$, the stabilizers of a 1 -face and of a 2 -face are both isomorphic to $2 \times D_{10}$, and the stabilizer of a 3 -face is isomorphic to $A_{5}$. Furthermore, $J_{1}$ acts transitively on the faces of each rank. Finally, we have that $\mathcal{Q}$ has 1463 vertices, 8778 edges, 87782 -faces and 2926 facets.

\subsection{The flags of $\mathcal{Q}$}

We want to show that the incidence defined above yields a regular polytope. To this end, we need to study the flags of $\mathcal{Q}$, together with their stabilizers in $G$. Recall that, 
in the language of incidence geometry, a flag is a set of pairwise incident element of $\Gamma$ (that is, a chain of an abstract polytope), while a chamber is a flag containing all the types (that is, a flag of an abstract polytope). Hence, we determine the sublattice of $\mathcal{Q}$ and use it to show that we have a thin regular residually connected incidence geometry, and hence an abstract regular polytope.

\subsubsection{Flags of rank 2}

We begin our study with flags of rank 2 , that is, pairs of incident elements of $\mathcal{Q}$. In particular, given an $i$-face $F_{i}$ we find out how many $j$-faces are incident to it. We base our arguments on the discussion on the Livingstone Graph in Sect. 3. Throughout, we also make use of counting combinatorial arguments; they can often be replaced by geometric graph arguments that although nicer, would make this paper longer than wanted.

Flags of type $\{0,1\}$ We fix $F_{0}:=\{a, \mathbb{P}\} \in \mathcal{F}_{0}$. There are 6 pentagons in $\mathbb{P} \cap \mathcal{W}$, that is, six options for $P \in F_{1}$. Once chosen $P \in \mathcal{W} \cap \mathbb{P}$, there are three pentagons in $\mathcal{O}$ associated to $P$, exactly one of them also belongs to $\mathbb{P}$, while the other two do not; we hence have two options to pick such a pentagon to be $P_{1} \in F_{0}$, and each choice will determine if $a=e_{0}$ or $a=e_{1}$ and hence completely determine $F_{1}$. Thus, there are 121 -faces $F_{1}$ incident to $F_{0}$.

On the other hand, if we start by fixing an $F_{1}=\left\{P, P_{1}, e,\left\{e_{0}, e_{1}\right\}\right\} \in \mathcal{F}_{1}$, we have that for an $F_{0}$ to be incident to $F_{1}$ there are two possibilities: either $a=e_{0}$ or $a=e_{1}$; each of these possibilities gives us a unique $\mathbb{P}$ and hence a unique 0 -face incident to $F_{1}$, implying that there are two 0 -faces incident to $F_{1}$.

Recall that $\operatorname{Stab}_{G} F_{0} \cong 2 \times A_{5}$, where the $A_{5}$ fixes the two vertices of $a$ while it acts transitively on the vertices, edges and pentagons in $\mathcal{W} \cap \mathbb{P}$ (resp., in $\mathcal{O} \cap \mathbb{P}$ ). Furthermore, by fixing $P \in \mathcal{W} \cap \mathbb{P}$, we also fix the associated pentagon in $\mathcal{O} \cap \mathbb{P}$, and hence the two edges $a^{\prime}, a^{\prime \prime}$ corresponding to the other two Petersen graphs spanned by $P$. The $2<\operatorname{Stab}_{G} F_{0}$ fixes the Petersen graph $\mathbb{P}$ pointwise, and it interchanges the two vertices of $a$, as well as the edges $a^{\prime}$ and $a^{\prime \prime}$. In particular, this implies that $\operatorname{Stab}_{G} F_{0}$ acts transitively on the 1-faces incident to $F_{0}$.

Since $G$ acts transitively on $\mathcal{F}_{0}$, we obtain that $G$ acts transitively on the flags of type $\{0,1\}$. Moreover, given a flag $\Omega_{0,1}:=\left\{F_{0}, F_{1}\right\}$ (of type $\{0,1\}$ ), the stabilizer of $\Omega_{0,1}$ is contained in both $2 \times D_{10} \cong \operatorname{Stab}_{G} F_{1}$ and $2 \times A_{5} \cong \operatorname{Stab}_{G} F_{0}$. In fact, since $\operatorname{Stab}_{G} F_{0}$ acts transitively on the 1 -faces incident to $F_{0}$, we have that

$$
\operatorname{Stab}_{G} \Omega_{0,1}=\operatorname{Stab}_{G} F_{0} \cap \operatorname{Stab}_{G} F_{1} \cong 2 \times D_{10} \cap 2 \times A_{5} .
$$

As the 2 in $\operatorname{Stab}_{G} F_{1}$ interchanges $e_{0}$ by $e_{1}$, it does not fix $F_{0}$ (similarly the 2 in $\operatorname{Stab}_{G} F_{0}$ does not fix $F_{1}$ ), we have that $\operatorname{Stab}_{G} \Omega_{0,1} \cong D_{10}$.

Flags of type $\{0,2\}$ Again, $G$ acts transitively on $\mathcal{F}_{0}$. Therefore, we can choose a 0 -face without loss of generality. When fixing a 0 -face $F_{0}=\{a, \mathbb{P}\}$, to see how many 2-faces are incident to $F_{0}$, we note that $\mathbb{P}$ has 15 edges, and each of them has two opposite edges. Choosing an edge $e^{\prime} \in \mathbb{P}$, let $b:=\{u, w\}$ and $b^{\prime}:=\left\{u^{\prime}, w^{\prime}\right\}$ be the two opposite edges in $\mathbb{P}$. Then $e^{\prime}$ is at distance two from all the vertices $u, w, u^{\prime}, w^{\prime}$. 


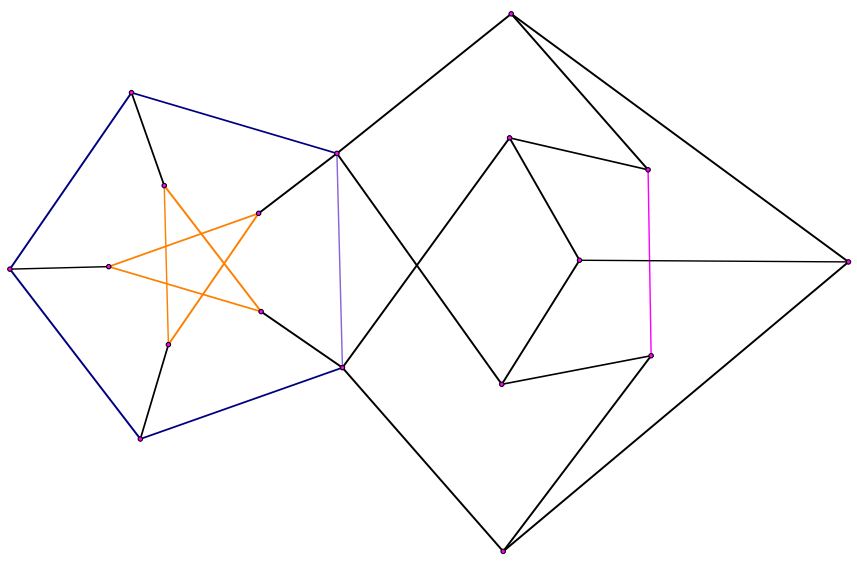

Fig. 6 The relation between the 0 - and the 2-faces of a flag of type $\{0,2\}$

From Sect. 3.1.3, we know that $e^{\prime}$ spans a white pentagon with two of these vertices, while it spans an orange pentagon with the other two; moreover, if $e^{\prime}$ spans a pentagon in $\mathcal{W}$ (resp., $\mathcal{O}$ ) with $u$, then it also spans a pentagon in $\mathcal{W}$ (resp., $\mathcal{O}$ ) with $w$. That is, only either $b$ or $b^{\prime}$ can be used as the edge of intersection of $\mathbb{P}$ with $P_{1}^{\prime} \in F_{2}$. Without loss of generality, let it be $b$, that is, the Petersen graph $\mathbb{P}_{0}^{\prime}$ associated to $e^{\prime}$ contains $b$. But we know that given an edge of a Petersen graph $\mathbb{P}_{0}^{\prime}$ it belongs to exactly two white pentagons of $\mathbb{P}_{0}^{\prime}$; in other words, for each $e^{\prime}$ we have a unique $\mathbb{P}_{0}^{\prime}$, but two option for $P_{1}^{\prime}$. Hence there are $15 \times 2=302$-faces incident to $F_{0}$.

Then, given a 2 -face, there are $\frac{30 \times\left|\mathcal{F}_{0}\right|}{\left|\mathcal{F}_{2}\right|}=\frac{30 \times 1463}{8778}=50$-faces incident to it.

Here, the $D_{10} \leq \operatorname{Stab}_{G} F_{2}$ acts as the symmetry group of $P_{1}^{\prime}$, always fixing $e^{\prime}$ pointwise, implying that $\operatorname{Stab}_{G} F_{2}$ acts transitively on the 0 -faces incident to $F_{2}$. And as $G$ acts transitively on $\mathcal{F}_{2}$ then it acts transitively on the flags of rank two of type $\{0,2\}$. Moreover, if $\Omega_{0,2}$ is a flag of type $\{0,2\}, \operatorname{Stab}_{G} \Omega_{0,2}=\operatorname{Stab}_{G} F_{0} \cap$ $\operatorname{Stab}_{G} F_{2} \cong 2^{2}$.

Flags of type $\{0,3\}$ Given a 0 -face $F_{0}=\{e, \mathbb{P}\}$, we count how many 3 -faces $\left\{v_{1}, V_{5}, V_{6}\right\}$ are incident to $F_{0}$. There are 10 options to choose $v_{1}$ from, namely the 10 vertices of $\mathbb{P}$. Now, the three neighbors of $v_{1}$ in $\mathbb{P}$ most be in $V_{6}$. By Sect. 3.2.2, since these three points are in both $N\left(v_{1}\right)$ and $\mathbb{P}$, they belong to exactly two blocks of $\Gamma$. That is, for each choice of $v_{1}$ there are two different possibilities of $F_{3}$ incident to $F_{1}$. Hence there are $10 \times 2=203$-faces incident to $F_{0}$.

Therefore, we have $\frac{20 \times\left|\mathcal{F}_{0}\right|}{\left|\mathcal{F}_{3}\right|}=\frac{20 \times 1463}{2926}=100$-faces incident to a given 3 -face.

To figure out what the stabilizer of a flag $\Omega_{0,3}=\left\{F_{0}, F_{3}\right\}$ is, note that $v_{1} \in \mathbb{P}$ and $\operatorname{Stab}_{G}\left\{v_{1}, \mathbb{P}\right\} \cong 2 \times S_{3}$, where the $2=:\langle\rho\rangle$ fixes $\mathbb{P}$ pointwise, but interchanges the vertices of its corresponding edge. Hence, $\rho$ fixes the 2 -arc $\left\{v_{0}, v_{1}, v_{2}\right\}$ (where $\left.v_{0}, v_{2} \in N\left(v_{1}\right) \cap \mathbb{P}\right)$, and thus the white pentagon $P_{\mathcal{W}}$ determined by it. Moreover, this implies that while $\rho$ fixes one of the Petersen graphs determined by $P_{\mathcal{W}}$, it swaps the other two of them. In other words, if $A, B$ and $C$ are the three blocks of $\Gamma$ determined by fixing $v_{1}$ and picking $v_{0}, v_{2} \in N\left(v_{1}\right)$, then $\rho$ fixes one of them and in- 
terchanges the other two. However, it does not fix any point of the fixed block. As $\rho$ fixes all three point in $N\left(v_{1}\right) \cap \mathbb{P}$, it cannot fix a block that contains these points. Hence $\operatorname{Stab}_{G} \Omega_{0,3} \cong S_{3}$.

Flags of type $\{1,2\} \quad$ Given $F_{1}=\left\{P, P_{1}, e,\left\{e_{0}, e_{1}\right\}\right\} \in \mathcal{F}_{1}$, each of the edges of $P$ can be chosen to be $e^{\prime} \in F_{2}$. Once $e^{\prime}$ is chosen, a Petersen graph $\mathbb{P}_{0}^{\prime}$ is determined. As we saw in Sect. 3, there is exactly one vertex, say $v_{0}$, in $\mathbb{P}_{0}^{\prime} \cap P$ and another vertex, say $u_{0}$, in $\mathbb{P}_{0}^{\prime} \cap P^{\prime}$. On the other hand, given an edge of a Petersen graph $\mathbb{P}_{0}^{\prime}$ and an edge $\left\{v_{0}, u_{0}\right\}$ of it there is exactly one pentagon $P^{\prime} \in \mathbb{P} \cap \mathcal{W}$ with $u_{0} \in P^{\prime}$ and $v_{0} \notin P^{\prime}$. This unique $P^{\prime}$ gives in turn a unique $P_{1}^{\prime} \in \mathcal{O}$ and hence determines a $F_{2}$ incident to $F_{1}$. That is, there are exactly five 2-faces incident to $F_{1}$. In turn, since $\left|\mathcal{F}_{1}\right|=\left|\mathcal{F}_{2}\right|$, given a 2 -face, there are five 1 -faces incident to it.

Moreover, $D_{10} \leq \operatorname{Stab}_{G}\left(F_{1}\right)$ acts transitively on the edges of $P$, implying that $\operatorname{Stab}_{G}\left(F_{1}\right)$ acts transitively on the 2 - faces incident to $F_{1}$. Since $G$ is transitive on $\mathcal{F}_{1}$, it acts transitively on the flags of type $\{1,2\}$.

The subgroup $\operatorname{Stab}_{G}\left(\Omega_{1,2}\right)$ is of index five in both $\operatorname{Stab}_{G}\left(F_{1}\right)$ and $\operatorname{Stab}_{G}\left(F_{2}\right)$ (where $\Omega_{1,2}=\left\{F_{1}, F_{2}\right\}$ ). The subgroup lattice of $J_{1}$ tell us that $\operatorname{Stab}_{G}\left(\Omega_{1,2}\right) \cong 2^{2}$.

Flags of type $\{1,3\}$ From Sect. 3.2.3, given a 3-face $F_{3}=\left\{v_{1}, V_{6}, V_{5}\right\}$, each two vertices of $V_{6}$ imply a unique choice of a third point $u$ to complete a Petersen graph in such a way that $u \in V_{5}$. Hence there are $\frac{6 \times 5}{2}=151$-faces incident to $F_{3}$. We then also have that there are $\frac{15 \times \mid \mathcal{F}_{3}}{\left|\mathcal{F}_{1}\right|}=53$-faces incident to a given 1 -face.

Note that inside $A_{5} \cong \operatorname{Stab}_{G} F_{3}$, the stabilizer of a set of two points of $V_{6}$ is isomorphic to $2^{2}$ and this subgroup stabilizes the point of $V_{5}$ that belongs to $P_{1}$ (see Sect. 3.2.3). That is, $\operatorname{Stab}_{G} \Omega_{1,3} \cong 2^{2}$, where $\Omega_{1,3}$ is a flag of type $\{1,3\}$.

Flags of type $\{2,3\} \quad$ From Sect. 3.2.4, given a 3-face $\left\{v_{1}, V_{6}, V_{5}\right\}$, there are six possibilities to choose $F_{2}$ incident to it, namely, one per edge with end points $v_{1}$ and a vertex of $V_{6}$. Moreover, given a 2-face $\left\{P^{\prime}, P_{1}^{\prime}, e^{\prime},\left\{e_{0}^{\prime}, e_{1}^{\prime}\right\}\right\}$, the choice of which vertex of $e^{\prime}$ is $v_{1}$ (and hence having the other vertex of $e^{\prime}$ belonging to $V_{6}$ ) completely determines a block in $N\left(v_{1}\right)$, and hence a $F_{3}$ incident to the given 2 -face. That is, there are exactly two 3-faces incident to a given 2-face.

Now, given $\Omega_{2,3}=\left\{F_{2}, F_{3}\right\}$ with $F_{2} \sim F_{3}$, we have that $\operatorname{Stab}_{G}\left[e^{\prime}\right] \cong D_{10}$ acts transitively not only on $P^{\prime}$ and $P_{1}^{\prime}$, but also on $V_{5}$ and $V_{6} \backslash\left\{v_{6}\right\}$ (where $e^{\prime}=\left\{v_{1}, v_{6}\right\}$ ). Hence, $\operatorname{Stab}_{G} \Omega_{2,3} \cong D_{10}$.

This discussion on flags of rank 2 is summarized in the following proposition.

Proposition 4.1 $G$ acts transitively on the flags of rank 2 of a given type of $\mathcal{Q}$. Furthermore, if $\Omega_{i, j}$ denotes a flag of type $\{i, j\}$, we have that

$$
\begin{aligned}
& \operatorname{Stab}_{G} \Omega_{0,1} \cong D_{10}, \quad \operatorname{Stab}_{G} \Omega_{0,2} \cong 2^{2}, \quad \operatorname{Stab}_{G} \Omega_{0,3} \cong S_{3}, \\
& \operatorname{Stab}_{G} \Omega_{1,2} \cong 2^{2}, \quad \operatorname{Stab}_{G} \Omega_{1,3} \cong 2^{2}, \quad \operatorname{Stab}_{G} \Omega_{2,3} \cong D_{10} .
\end{aligned}
$$

\subsubsection{Flags of rank 3 and chambers of $\mathcal{Q}$}

We now prove that every flag is contained in a chamber, and hence the incidence structure $\mathcal{Q}$ is, in fact, a geometry; to do so, we first show that every rank 2 flag 
Fig. 7 A flag $\left\{F_{0}, F_{1}\right\}$ of type $\{0,1\}$ and elements to construct a 2-face incident to $F_{1}$

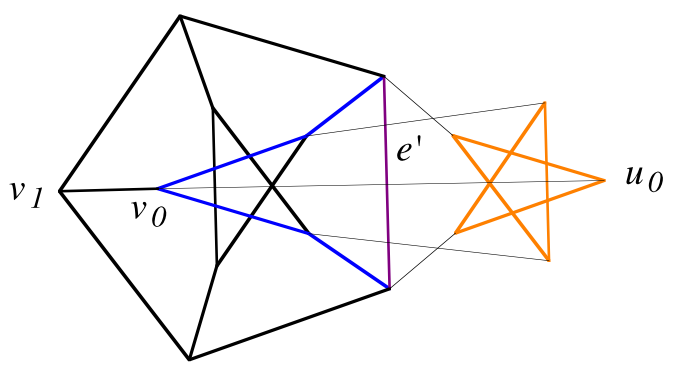

is contained in rank 3 flags. Moreover, from the arguments we give, together with some given above, it is also clear that such geometry is thin. Throughout, for each $J \subseteq\{0,1,2,3\}, \Omega_{J}$ denotes a rank $|J|$ flag of $\mathcal{Q}$ of type $J$.

For each case, we take a rank 2 flag $\Omega_{i, j}$, extend it to a rank 3 flag $\Omega_{i, j, k}$ and show that $\operatorname{Stab}_{G} \Omega_{i, j}$ acts transitively on the $k$-faces incident to it. In this way, we obtain that $G$ is transitive on the flags of type $\{i, j, k\}$. In turn, this immediately implies that every flag of type $\{i, k\}$ or $\{j, k\}$ can be extended to one of type $\{i, j, k\}$.

In a similar way, to show that every flag is contained in a chamber, it is enough to show that a flag $\Omega_{0,1,2}$ is contained in a chamber and that $\operatorname{Stab}_{G} \Omega_{0,1,2}$ acts transitively on the 3 -faces incident to such rank 3 flag.

Flags of type $\{0,1,2\}$ Let us start with a rank 2 flag $\Omega_{0,1}=\left\{F_{0}, F_{1}\right\}$ of type $\{0,1\}$, with $F_{0}=\{a, \mathbb{P}\}$ and $F_{1}=\left\{P, P_{1}, e,\left\{e_{0}, e_{1}\right\}\right\}$ (so that $P \in \mathbb{P}$ and $a \in\left\{e_{0}^{\prime}, e_{1}^{\prime}\right\}$ ) and let $\mathbb{P}_{0}$ be the Petersen graph spanned by $P$ and $P_{1}$.

Let $F_{2} \in \mathcal{F}_{2}$ be such that $F_{2} \sim F_{1}$, with $F_{2}=\left\{P^{\prime}, P_{1}^{\prime}, e^{\prime},\left\{e_{0}^{\prime}, e_{1}^{\prime}\right\}\right\}$. Thus, $e^{\prime} \in P$ and $\left|V\left(P \cap P_{1}^{\prime}\right)\right|=\left|V\left(P^{\prime} \cap P_{1}\right)\right|=1$. We shall show that $F_{2} \sim F_{0}$, and so any flag of type $\{0,1\}$ can be completed to one of type $\{0,1,2\}$. In other words, we need to show that $e^{\prime} \in \mathbb{P}$ and $P_{1}^{\prime} \cap \mathbb{P}$ is an edge opposite to $\mathbb{P}$.

We have that $e^{\prime} \in P$, and so $e^{\prime} \in \mathbb{P}$. Moreover, note that if $v_{0} \in P, u_{0} \in P_{1}$ are adjacent vertices that, together with $e^{\prime}$, span pentagons in $\mathcal{W}$, then $\left\{v_{0}, u_{0}\right\}$ is an edge opposite to $e^{\prime}$ in $\mathbb{P}_{0}$. Since $F_{2} \sim F_{1},\left\{v_{0}, u_{0}\right\} \in \mathbb{P}_{0}^{\prime}$, the Petersen graph spanned by $P^{\prime}$ and $P_{1}^{\prime}$. Now, in $\mathbb{P}, v_{0}$ and $e^{\prime}$ span a pentagon in $\mathcal{W}$; call $v_{1}$ the other vertex of $\mathbb{P}$ that also spans a pentagon in $\mathcal{W}$ with $e^{\prime}$ (see Fig. 7). Then $v_{1} \in \mathbb{P}_{0}^{\prime}$, and further $\left\{v_{0}, v_{1}\right\}$ is an edge of $\mathbb{P}$. Note that since $F_{2} \sim F_{1}$, we have $v_{0} \in P_{1}^{\prime}$ and $u_{0} \in P^{\prime}$. Hence, to show that $F_{2} \sim F_{0}$, we need to show that $v_{1} \in P_{1}^{\prime}$. To this end, we observe that $P^{\prime}$ and $P_{1}^{\prime}$ partition the vertices of $\mathbb{P}_{0}^{\prime}$, and given a vertex in $P^{\prime}$ (resp., $P_{1}^{\prime}$ ), one of its neighbors is in $P_{1}^{\prime}$ (resp., $P^{\prime}$ ), while the other two are in $P^{\prime}$ (resp., $P_{1}^{\prime}$ ). We then have that $v_{0} \in P_{1}^{\prime}$ and $v_{1} \neq u_{0}$ are both neighbors of $v_{0}$; furthermore, we know that $u_{0} \in P^{\prime}$, implying that $v_{1} \in P_{1}^{\prime}$, and hence every flag of type $\{0,1\}$ is contained in a flag of type $\{0,1,2\}$.

Now, given faces $F_{0} \sim F_{2}$, we shall find the 1-faces $F_{1}$ incident to this flag. To this end, we shall determine the pentagons $P$ and $P_{1}$. The incidences $F_{0} \sim F_{1} \sim F_{2}$ imply that $e^{\prime}$ and the vertex $v_{0}\left(=P_{1}^{\prime} \cap P\right)$ determine $P$. Although $e^{\prime}$ is given by $F_{2}$, we have two different possibilities to choose $v_{0}$ from, namely the two vertices of the intersection $\mathbb{P} \cap P_{1}^{\prime}$. Following arguments similar to those above, one can see that once $v_{0}$ is chosen (and hence $P$ is determined), there is a unique $P_{1}$ that completes 
Fig. 8 A flag $\left\{F_{0}, F_{2}\right\}$ of type $\{0,2\}$ and elements to construct a 1-face incident to them

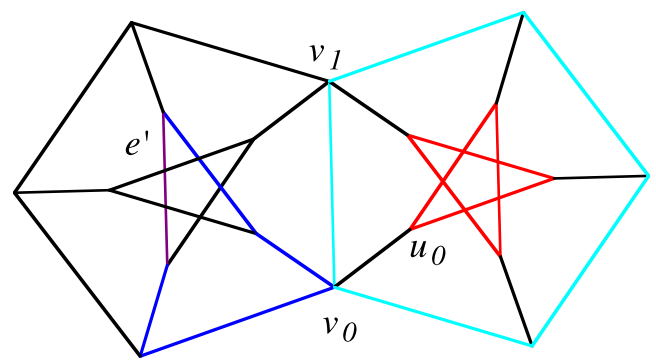

$F_{1}$ as wanted (see Fig. 8). That is, there are exactly two 1-faces incident to a given flag of type $\{0,2\}$.

To find the stabilizer of the flag $\Omega_{0,1,2}=\left\{F_{0}, F_{1}, F_{2}\right\}$, we note that $\operatorname{Stab}_{G}\left[\Omega_{0,2}\right] \cong$ $2^{2}$. If $\rho, \tau$ are the generators of this $2^{2}$, then one of them, say $\rho$, interchanges the vertices of $e^{\prime}$, and fixes $\mathbb{P}_{0}^{\prime}$ pointwise; while the other one, say $\tau$, fixes $e^{\prime}$ pointwise and acts on $\mathbb{P}_{0}^{\prime}$ as the reflection that interchanges $v_{0}$ and $v_{1}$. Hence, $\rho \in \operatorname{Stab}_{G}\left[\Omega_{0,1,2}\right]$, while $\tau$ interchanges the two 1 -faces incident to $\Omega_{0,2}$. That is, $\operatorname{Stab}_{G}\left[\Omega_{0,2}\right]$ acts transitively on the 1 -faces incident to $\Omega_{0,2}$. On the other hand, we know that $G$ acts transitively on the flags of type $\{0,2\}$, implying that $G$ acts transitively on the flags of type $\{0,1,2\}$. Furthermore, $\operatorname{Stab}_{G}\left[\Omega_{0,1,2} \cong 2\right.$.

Flags of type $\{1,2,3\}$ Given $F_{1} \sim F_{3}$, to have an $F_{2}$ incident to both we choose $e^{\prime}$ as one of the two edges of $P$ that contain $v_{1}$. By the discussion on flags of type $\{1,2\}$ the choice of such $e^{\prime}$ completely determines $F_{2}$. Hence there are exactly two 2-faces incident to a flag of type $\{1,3\}$.

It is now clear that starting with $\operatorname{Stab}_{G} \Omega_{1,3} \cong 2^{2}$, there is only one involution that fixes $e^{\prime}$, implying that $\operatorname{Stab}_{G} \Omega_{1,2,3} \cong 2$ (the intersection between the pointwise stabilizer of the two points in $N\left(v_{1}\right)$ that determine three blocks, and the stabilizer of one such block).

Flags of type $\{0,1,3\}$ Given faces $F_{0} \sim F_{1}$ and $F_{1} \sim F_{3}$, it is now straightforward from Sect. 3.2.2 to see that $F_{0} \sim F_{3}$. Furthermore, the stabilizer of a flag $\Omega_{0,1,3}$ of type $\{0,1,3\}$ corresponds to the elements of $\operatorname{Stab}_{G} \Omega_{0,1} \cong D_{10}$ that fix a point of the pentagon $P$. That is, $\operatorname{Stab}_{G} \Omega_{0,1,3} \cong 2$.

Flags of type $\{0,2,3\}$ If we now take faces $F_{0} \sim F_{2}$ and $F_{2} \sim F_{3}$, it is immediate that $v_{1} \in \mathbb{P}$ and that if $e^{\prime}=\left\{v_{1}, u\right\}, u \in V_{6}$. By the discussion of the flags of type $\{0,1,2\}$, we can observe further that the other two vertices in $N\left(v_{1}\right) \cap \mathbb{P}$ are also in $V_{6}$, implying that $F_{0} \sim F_{3}$.

Since the stabilizer of the flag $\Omega_{0,2}$, isomorphic to $2^{2}$, allows us to swap the vertices of $e^{\prime}$, while $\operatorname{Stab}_{G} \Omega_{0,2,3}$ does not contain such element, but does contain the one that is a reflection on the edge $P_{1}^{\prime} \cap \mathbb{P}$, the subgroup $\operatorname{Stab}_{G} \Omega_{0,2,3} \cong 2$.

We summarize the above discussion in the following proposition. 
Fig. 9 The subgroup lattice of $\mathcal{Q}$

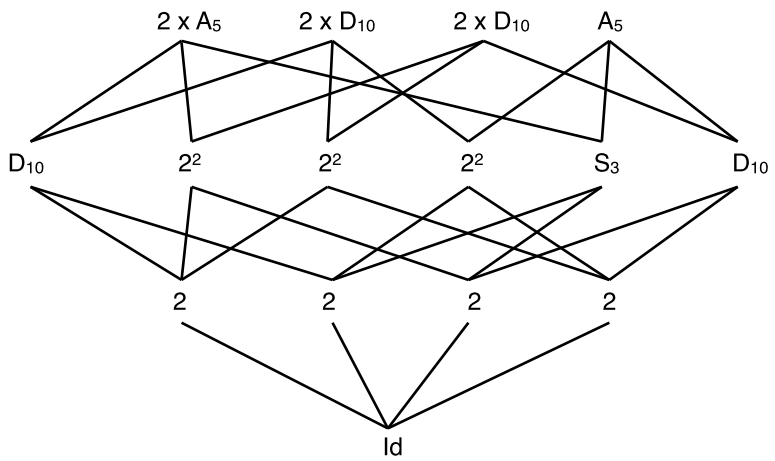

Proposition 4.2 $\mathcal{Q}$ is transitive on flags of rank 3 of each type. Furthermore, if $\Omega_{i, j, k}$ denotes a flag of type $\{i, j, k\}$, we have that

$$
\operatorname{Stab}_{G} \Omega_{0,1,2} \cong \operatorname{Stab}_{G} \Omega_{1,2,3} \cong \operatorname{Stab}_{G} \Omega_{0,1,3} \cong \operatorname{Stab}_{G} \Omega_{0,2,3} \cong 2 .
$$

Chambers Given a flag $\Omega_{0,1,3}=\left\{F_{0}, F_{1}, F_{3}\right\}$ of type $\{0,1,3\}$, we proceed as we did for flags of type $\{1,2,3\}$ and obtain that there are exactly two $F_{2}$ 's incident to $\Omega_{0,1,3}$. Furthermore, $\operatorname{Stab}_{G} \Omega_{0,1,3} \cong 2$ is generated precisely by the 'reflection' of $P$ that interchanges the two possible choices for $e^{\prime}$ that determine $F_{2}$. This implies that $\operatorname{Stab}_{G} \Omega_{0,1,3}$ is transitive on the 2-faces incident to $\Omega_{0,1,3}$ and also that $\operatorname{Stab}_{G} \Omega_{0,1,2,3}$ is the identity.

From this we can then see that the sublattice for $\mathcal{Q}$ is as in Fig. 9.

Theorem 4.1 The incidence structure $\mathcal{Q}$ is a thin and residually connected geometry. Its automorphism group $G$ is isomorphic to $J_{1}$. Moreover, $G$ acts flag-transitively and regularly on $\mathcal{Q}$. Finally, the Buekenhout diagram of $\mathcal{Q}$ is as follows.

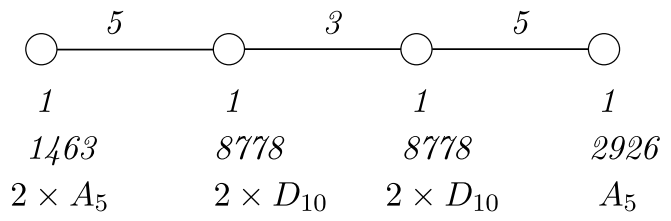

Proof This theorem is an immediate consequence of Theorem 2.1, the sublattice given in Fig. 9 and the fact that $J_{1}$ is the automorphism group of the Livingstone graph.

\section{A polytope of type $\{5,6,5\}$}

We finish by defining a second incidence geometry of rank 4 arising from the Livingstone graph. The vertices, edges and 2-faces of this new geometry $\mathcal{P}$ are defined in the same way as those for the $\{5,3,5\}$, and we now change only the 3 -faces. Since the details that show that the given construction is indeed a regular polytope of rank 4 
Fig. 10 The subgroup lattice of $\mathcal{P}$

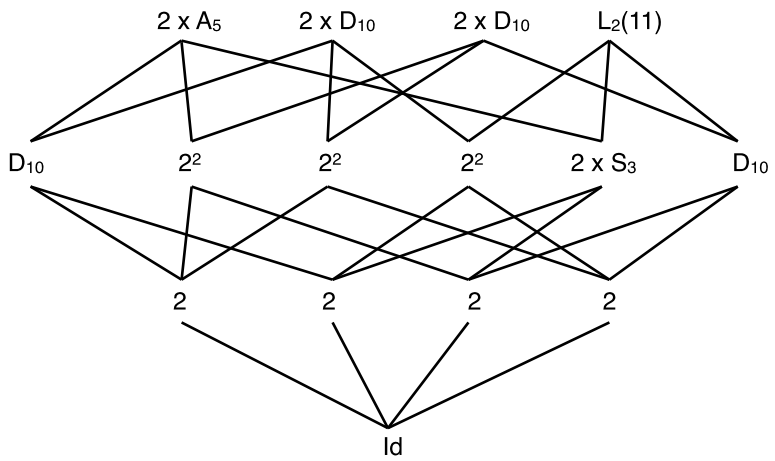

with automorphism group isomorphic to $J_{1}$ are similar to the ones given in the above section, for space reasons, we leave them out.

We start by defining the faces (of $\mathcal{P}$ ) of each rank as follows:

$$
\begin{aligned}
& \mathcal{F}_{0}(\mathcal{P}):=\{\{e, \mathbb{P}\} \mid e \in E(\mathcal{G}), \mathbb{P} \text { is the Petersen subgraph associated to } e\}, \\
& \mathcal{F}_{1}(\mathcal{P}):=\mathbb{P}(\mathcal{G}), \quad \mathcal{F}_{2}(\mathcal{P}):=\mathbb{P}(\mathcal{G}), \quad \mathcal{F}_{3}(\mathcal{P}):=V(\mathcal{G}) .
\end{aligned}
$$

Although the edges and 2-faces of $\mathcal{P}$ are defined using the same set of elements as in Sect. 4, the incidences of elements of these sets will be defined in a different way. In what follows, let $F_{i}$ represent an element of $\mathcal{F}_{i}(\mathcal{P}), i=0,1,2,3$. To avoid confusion, an element $F_{1} \in \mathcal{F}_{1}(\mathcal{P})$ will be denoted as $\left\{P, P_{1}, e,\left\{e_{0}, e_{1}\right\}\right\}$ while an element $F_{2} \in \mathcal{F}_{2}(\mathcal{P})$ will be denoted with primes, that is, $F_{2}=\left\{P^{\prime}, P_{1}^{\prime}, e^{\prime},\left\{e_{0}^{\prime}, e_{1}^{\prime}\right\}\right\}$. As each $a \in E(\mathcal{G})$ has a Petersen graph associated to it, an element of $\mathcal{F}_{0}(\mathcal{P})$ can be thought of as an edge of $\mathcal{G}$, a Petersen graph $\mathbb{P}$, or an edge with its respective Petersen graph.

Let $\sim$ denote the incidence relation between faces. We shall say that:

$$
\begin{aligned}
& F_{3} \sim F_{0} \quad \Leftrightarrow \quad F_{3} \text { is at distance two from } F_{0} \text { and they span a pentagon in } \mathcal{W} ; \\
& F_{3} \sim F_{1} \quad \Leftrightarrow \quad F_{3} \in P \in F_{1} ; \\
& F_{3} \sim F_{2} \quad \Leftrightarrow \quad F_{3} \in e^{\prime} \in F_{2} ; \\
& F_{2} \sim F_{0} \quad \Leftrightarrow \quad e^{\prime} \in \mathbb{P}, \quad P^{\prime} \cap \mathbb{P} \text { is an edge } \\
& F_{2} \sim F_{1} \quad \Leftrightarrow \quad e^{\prime} \in P, \quad\left|V\left(P \cap P^{\prime}\right)\right|=\left|V\left(P_{1} \cap P_{1}^{\prime}\right)\right|=1 ; \\
& F_{1} \sim F_{0} \quad \Leftrightarrow \quad P \in \mathbb{P} \text { and } a \in\left\{e_{0}, e_{1}\right\} .
\end{aligned}
$$

Note that from the definition of our faces, and the results from Sect. 3, we have that $\mathcal{P}$ has 14630 -faces, and the stabilizer of each of them is $2 \times A_{5} ; 87781$-faces, each with stabilizer $2 \times D_{10}$; also 8778 2-faces with stabilizers $2 \times D_{10}$; and 266 3-faces, each with stabilizer $L_{2}(11)$. Moreover, $G$ acts transitively on the faces of $\mathcal{P}$ of each rank. In fact, each $\gamma \in G$ can be seen as an automorphism of $\mathcal{P}$, that is, a bijection of the faces of $\mathcal{P}$ that preserves the incidences and hence the rank.

Proposition 5.1 The incidence structure $\mathcal{P}, \sim$ is an incidence geometry with sublattice given in Fig. 10. 


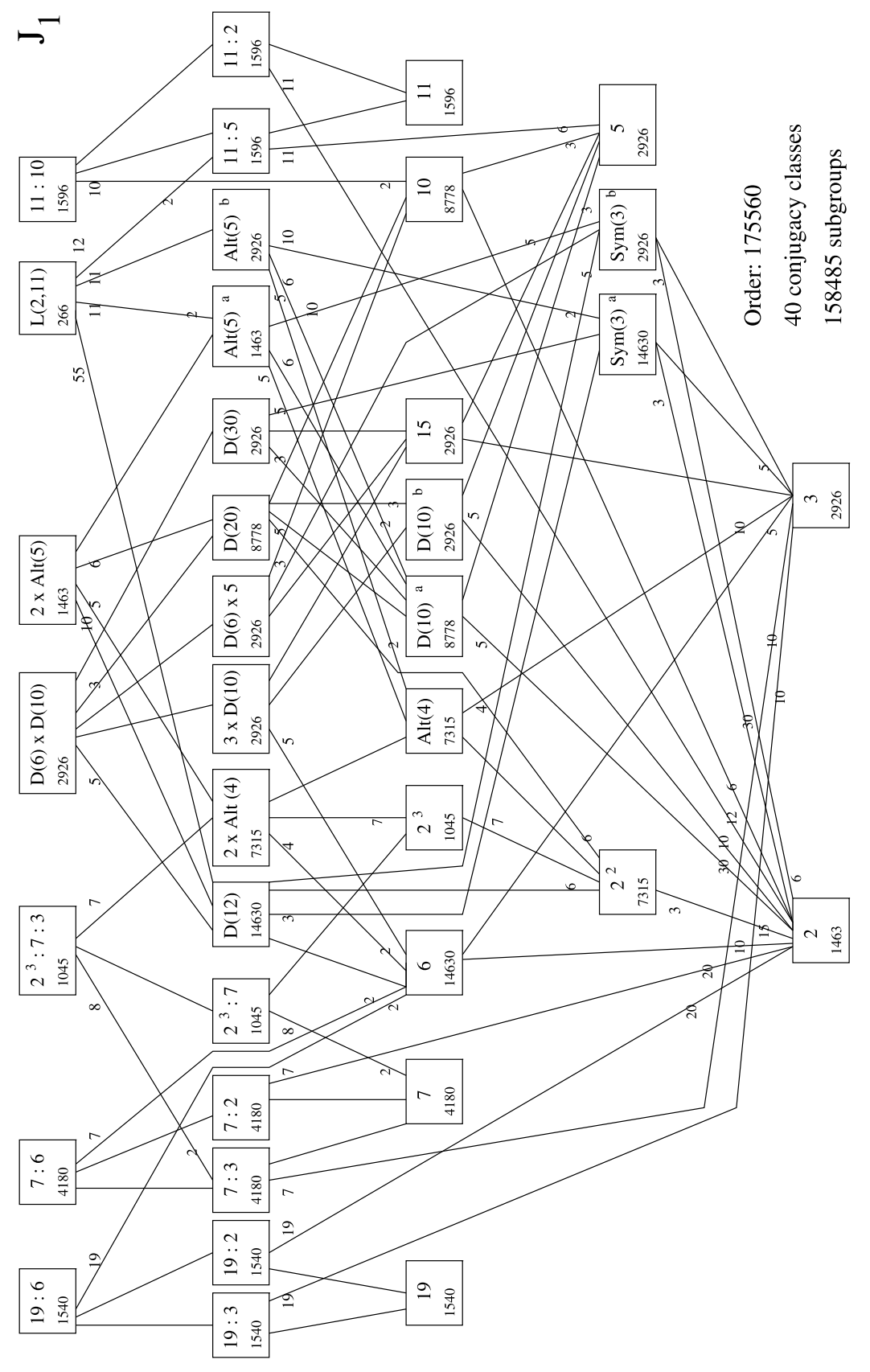

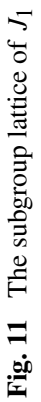


Theorem 5.1 The incidence structure $\mathcal{P}$ is a thin and residually connected geometry. Its automorphism group $G$ is isomorphic to $J_{1}$. Moreover, $G$ acts flag-transitively and regularly on $\mathcal{P}$. Finally, the Buekenhout diagram of $\mathcal{P}$ is as follows.

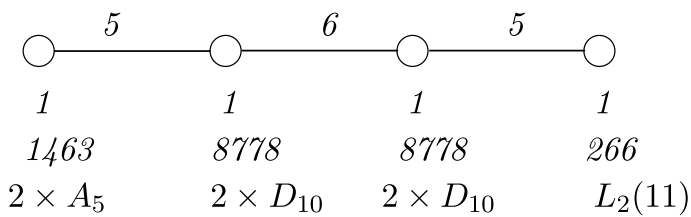

Acknowledgements Research of I. Hubard was supported by Sociedad Matemática MexicanaFundación Sofía Kovalevskaia.

\section{References}

1. Aschbacher, M.: Flag structures on Tits geometries. Geom. Dedic. 14, 21-31 (1983)

2. Brouwer, A.E., Cohen, A.M., Neumaier, A.: Distance-Regular Graphs. Springer, Berlin (1989)

3. Buekenhout, F.: The geometry of the finite simple groups. In: Rosati, L.A. (ed.) Buildings and the Geometry of Diagrams. Lect. Notes in Math., vol. 1181, pp. 1-78. Springer, Berlin (1986)

4. Buekenhout, F. (ed.): Handbook of Incidence Geometry. Buildings and Foundations. Amsterdam, Elsevier (1995)

5. Buekenhout, F., Cara, P., Vanmeerbeek, K.: Geometries of the group PSL(2, 11). Geom. Dedic. 83, 169-206 (2000)

6. Gottschalk, H., Leemans, D.: The residually weakly primitive geometries of the Janko group $\mathrm{J}_{1}$. In: Groups and Geometries, pp. 65-79. Birkhäuser, Basel (1998)

7. Hartley, M.I., Leemans, D.: Quotients of a universal locally projective polytope of type $\{5,3,5\}$. Math. Z. 247(4), 663-674 (2004)

8. Hartley, M.I., Leemans, D.: On thin regular geometries of rank four for the Janko group $J_{1}$. Innov. Incid. Geom. 1, 181-190 (2005)

9. Hartley, M.I., Leemans, D.: A new Petrie-like construction for abstract polytopes. J. Comb. Theory, Ser. A 115(6), 997-1007 (2008)

10. Janko, Z.: A new finite simple group with abelian 2-Sylow subgroups. Proc. Natl. Acad. Sci. USA 53, 657-658 (1965)

11. Janko, Z.: A new finite simple group with abelian Sylow 2-subgroups and its characterization. J. Algebra 3, 147-186 (1966)

12. Lefèvre-Percsy, C., Percsy, N., Leemans, D.: New geometries for finite groups and polytopes. Bull. Belg. Math. Soc. Simon Stevin 7(4), 583-610 (2000)

13. Livingstone, D.: On a permutation representation of the Janko group. J. Algebra 6, 43-55 (1967)

14. McMullen, P., Schulte, E.: Abstract Regular Polytopes. Encyclopedia of Mathematics and Its Applications, vol. 92. Cambridge University Press, Cambridge (2002)

15. Pasini, A.: Diagram Geometries. Oxford University Press, London (1994)

16. Perkel, M.: A characterization of $J_{1}$ in terms of its geometry. Geom. Dedic. 9(3), 291-298 (1980)

17. Schönert, M., et al:: GAP: Groups, algorithms and programming. Lehrstuhl D für Mathematik, Aachen (1994)

18. Schulte, E.: Reguläre Inzidenzkomplexe II. Geom. Dedic. 14, 33-56 (1983)

19. J. Tits. Géométries polyédriques et groupes simples. Atti 2a Riun. Group. Math. Express. Lat. Firenze, 66-88 (1962) 